\title{
Zurückhaltendes Urteil mit weitreichenden Folgen: Bundesgericht erklärt Bündner Wahlsystem für verfassungswidrig
}

Bemerkungen zum Bundesgerichtsurteil 1C_495/2017vom 29. Juni 2019

Marco Ehrat *

Das kürzlich ergangene Bundesgerichtsurteil zum Bündner Parlamentswahlsystem ist das jüngste in einer Reihe von Entscheiden, in denen das Bundesgericht seit 2002 seine Rechtsprechung zu kantonalen Wahlsystemen weiterentwickelt und reformiert hat. Mit der Beschwerde wurde auch eine langjährige innerkantonale Debatte über das Wahlsystem auf Bundesebene getragen. Das Bundesgericht beschäftigte sich erstmals mit einem «reinen Majorzsystem», wobei es die Rechtsprechung zu gemischten Wahlsystemen (Proporz/Majorz) weitgehend auf reine Majorzsysteme übertrug - und eine erstaunlich tiefe Hürde für deren Verfassungskonformität aufstellte. Doch auch diesen Anforderung genügt das Bündner Wahlsystem nicht.

I. Prozessgeschichte und Erwägungen des Bundesgerichts ................................ 18

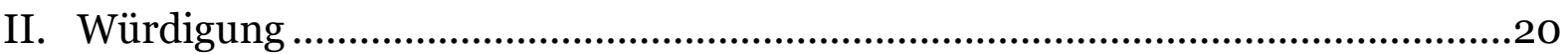

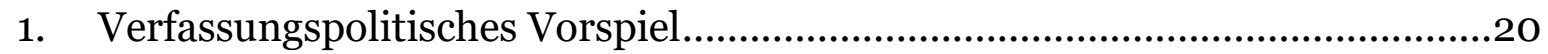

2. Zulässigkeit der Überprüfung der Kantonsverfassung................................... 21

3. Die Hauptprobleme des Bündner Wahlsystems.........................................22

4. Mögliche Folgen des Entscheids ............................................................26

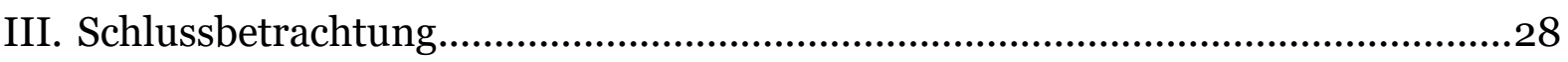

Zitiervorschlag: Marco Ehrat, Zurückhaltendes Urteil mit weitreichenden Folgen: Bundesgericht erklärt Bündner Wahlsystem für verfassungswidrig, in: sui-generis 2020, S. 17

URL: $\quad$ sui-generis.ch/117

DOI: $\quad$ https://doi.org/10.21257/sg.117

* Marco Ehrat, MLaw, Wissenschaftlicher Mitarbeiter beim Sekretariat der Wettbewerbskommission, Bern (ehrat.marco@gmail.com). Für die kritische Durchsicht des Beitrags und die zahlreichen wertvollen Hinweise dankt der Autor Herrn Prof. Dr. Andreas Glaser und Herrn MLaw David Eschle.

Dieses Werk ist lizenziert unter einer Creative Commons Namensnennung - Weitergabe unter gleichen Bedingungen 4.o International Lizenz. 


\section{Prozessgeschichte und Erwägungen des Bundesgerichts}

1 Im Vorfeld der Grossratswahlen von 2018 verteilte die Bündner Regierung im September 2017 die Sitze auf die Wahlkreise. ${ }^{1}$ Dagegen führten 54 Private und fünf politische Parteien Beschwerde sowohl vor dem kantonalen Verwaltungsgericht in seiner Funktion als Verfassungsgericht als auch vor Bundesgericht. ${ }^{2}$ Die Beschwerdeführer forderten, dass die zuständigen Behörden des Kantons in einem Appellentscheid aufzufordern seien, das Wahlsystem verfassungskonform zu gestalten. Nachdem das Verwaltungsgericht Graubünden einen Nichteintretensentscheid gefällt hatte, 3 trat das Bundesgericht auf die Sache ein und fällte einen teilweise gutheissenden Entscheid. Die Beschwerden stellten nach 2014 den zweiten Versuch dar, das Bündner Wahlsystem für verfassungswidrig erklären zu lassen. 4

Urteil setzt sich wie folgt zusammen: Nach Ausführungen zu den Eintretensvoraussetzungen, insbesondere zum Nichteintretensentscheid des Bündner Verwaltungsgerichts (E. 1), beschreibt das Bundesgericht das Bündner Wahlsystem

1 Regierungsmitteilung vom 14. September 2017 (Kanton Graubünden).

2 Verfasser der Beschwerde war der kürzlich verstorbene Prof. Dr. Andreas Auer, namhafter Kritiker von Majorzsystemen. Vgl. dazu Andreas Auer, Staatsrecht der schweizerischen Kantone, Bern 2016, Rz 1131 ff. m.w.H.

3 Urteil des Verwaltungsgerichts Graubünden V 175 vom 16. Januar 2018. Das Verwaltungsgericht trat auf die Beschwerde nicht ein, weil es dem Rechtsvertreter der Beschwerdeführer an der Postulationsfähigkeit fehlte (E. 6). Ausserdem verneinte das Verwaltungsgericht seine Kompetenz, Bestimmungen der Kantonsverfassung auf ihre Vereinbarkeit mit Bundesrecht zu überprüfen (E. 7).

4 Vgl. Urteil des Verwaltungsgerichts Graubünden V 1410 vom 17. Dezember 2015.
(E. 3.1-3.4) und wiederholt die Bedenken bezüglich dieses Systems anlässlich der Gewährleistung der KV/GR5 2004 (E. 3.5). Es folgen Erwägungen zu den Anforderungen an kantonale Parlamentswahlen (E. 4) sowie zur Zulässigkeit der inzidenten Kontrolle von Normen in Kantonsverfassungen (E. 5). Schliesslich setzt sich das Bundesgericht mit den Rügen der Beschwerdeführer zur Sitzverteilung auf die Wahlkreise (E. 6), zur Stimmkraftgleichheit (E. 7) und zur Erfolgswertgleichheit (E. 8) auseinander.

3 Neben der Verletzung der Bundesverfassung ${ }^{6}$ durch das GRG/GR7 rügen die Beschwerdeführer auch die Verfassungswidrigkeit von Art. 27 Abs. 2 und 4 KV/GR. ${ }^{8}$ Das Bundesgericht setzt sich zunächst mit der Frage auseinander, ob es berechtigt ist, diese Bestimmungen der Kantonsverfassung auf ihre Konformität mit der Bundesverfassung zu prüfen, ob es also eine inzidente Normenkontrolle vornehmen darf. Nach gefestigter Praxis überprüft das Bundesgericht Bestimmungen von Kantonsverfassungen nur, «wenn das übergeordnete Recht im Zeitpunkt der Gewährleistung noch nicht in Kraft war oder sich seither in einer Weise weiterentwickelt hat, der es Rechnung zu tragen gilt». 9 Diese Voraussetzungen erachtet das Bundesgericht in Bezug auf Art. 27 Abs. 2 KV/GR als erfüllt, zumal sich das Wahlrecht durch die verschiedenen höchstrichterlichen Urteile seit 2002

5 Verfassung des Kantons Graubünden vom 14. September 2003 (KV/GR; BR 110.100).

6 Bundesverfassung der Schweizerischen Eidgenossenschaft vom 18. April 1999 (BV; SR 101).

7 Gesetz über den Grossen Rat (Grossratsgesetz) vom 8. Dezember 2005 (BR 170.100; nachfolgend: GRG/GR).

8 Urteil des Bundesgerichts 1C_495/2017 vom 29. Juli 2019 (zur Publikation vorgesehen) E. 5.

9 Ebenda, E. 5.1; BGE 142 I 99 E. 4.3.3, S. 117. 
wesentlich verändert habe. ${ }^{10}$ Daher überprüft das Bundesgericht neben den kantonalen gesetzlichen Bestimmungen zum Wahlrecht auch die entsprechenden Verfassungsbestimmungen ${ }^{11}$.

In Erwägung 6 setzt sich das Bundesgericht mit der Rüge auseinander, die Vorschriften zur Verteilung der Sitze auf die Wahlkreise verletzten die Rechtsgleichheit. Diesbezüglich fordern die Beschwerdeführer, dass die Sitze entsprechend der gesamten Wohnbevölkerung verteilt werden und nicht nur gestützt auf die Schweizer Wohnbevölkerung (vgl. Art. 27 Abs. 4 KV/GR). ${ }^{12}$ Das Bundesgericht liess die Frage der Zulässigkeit einer Normenkontrolle offen, da es ohnehin keinen Anlass sah, seine bisherige Rechtsprechung zu überdenken. Es ist also den Kantonen weiterhin erlaubt, für die Sitzverteilung auf die gesamte Wohnbevölkerung, die schweizerische Wohnbevölkerung oder auf die Stimmberechtigten abzustellen.

Urteil des Bundesgerichts 1C_495/2017 vom 29. Juli 2019 (zur Publikation vorgesehen) E. 5.4; vgl. zur Entwicklung der Rechtsprechung Nagihan Musliu, Die verfassungsrechtlichen Anforderungen an die Wahlsysteme der Kantone, in: Andreas Glaser (Hrsg.), Das Parlamentswahlrecht der Kantone, Zürich/St. Gallen 2018, 1-32, insb. Rz 32 ff.; vgl. auch Anina Weber, Schweizerisches Wahlrecht und die Garantie der politischen Rechte, Eine Untersuchung ausgewählter praktischer Probleme mit Schwerpunkt Proporzwahlen und ihre Vereinbarkeit mit der Bundesverfassung, Zürich 2016, Rz 312 ff., 362 ff.

11 I.c. Art. 27 Abs. 2 KV/GR, bezüglich Abs. 4 wurde die Überprüfungsbefugnis offengelassen (vgl. sogleich Rz 4): Urteil des Bundesgerichts 1C_495/2017 vom 29. Juli 2019 (zur Publikation vorgesehen) E. 5.5.

12 Urteil des Bundesgerichts 1C_495/2017 vom 29. Juli 2019 (zur Publikation vorgesehen) E. 6; zu den Modalitäten der Sitzverteilung für die Grossratswahlen in Graubünden vgl. Marco Ehrat/Julia Eigenmann, Kantone mit Mehrheitswahlverfahren, in: Andreas Glaser (Hrsg.), Das Parlamentswahlrecht der Kantone, Zürich/ St. Gallen 2018, 171-203, Rz 10.
Die Verteilung der Sitze auf die Bündner Wahlkreise anhand der Schweizer Wohnbevölkerung erachtet das Bundesgericht somit als verfassungskonform. ${ }^{13}$

$5 \quad$ In der folgenden Erwägung 7 prüft das Bundesgericht eine Verletzung der Stimmkraftgleichheit. ${ }^{14}$ Es stellt den neuen Richtwert auf, dass Abweichungen der Wahlkreisgrösse von bis zur Hälfte der durchschnittlichen Repräsentationsziffer ${ }^{15}$ aller Wahlkreise grundsätzlich rechtfertigbar seien. Wahlkreise, die wie z.B. Avers höhere Abweichungen aufweisen, seien nicht mehr verfassungskonform, zumal sie sich auch nicht mehr auf ihre Autonomie berufen könnten. ${ }^{16}$

6 Schliesslich setzt sich das Bundesgericht mit einer möglichen Verletzung der Erfolgswertgleichheit auseinander. Dabei geht es auf die dem Mehrheitswahlverfahren inhärenten Probleme der Verwirklichung der Erfolgswertgleichheit ein und hält fest, dass die Parteizugehörigkeit auch in solchen Wahlsystemen von Bedeutung sei. ${ }^{17}$ Daher sei es problematisch, dass die realen Parteistärken nicht im Parlament widergespiegelt würden. All dies bedeute aber noch nicht, dass das System verfassungswidrig sei. ${ }^{18}$ Der Erfolgswertgleichheit sei in reinen Majorz-

13 Vgl. Urteil des Bundesgerichts 1C_495/2017 vom 29. Juli 2019 (zur Publikation vorgesehen) E. $6.1 \mathrm{ff}$.

$14 \mathrm{Zu}$ den drei Teilgehalten der Wahlrechtsgleichheit (Zählwertgleichheit, Stimmkraftgleichheit, Erfolgswertgleichheit) vgl. BGE 140 I 394 E. 8.3, S. 402 f.; BGE 129 I 185 E. 7.3, S. 199 f.

15 Die Repräsentationsziffer entspricht der Division der gesamten schweizerischen Wohnbevölkerung durch die total zu vergebenden Sitze (Urteil des Bundesgerichts 1C_495/2017 vom 29. Juli 2019 [zur Publikation vorgesehen] E. 7).

16 Urteil des Bundesgerichts 1C_495/2017 vom 29. Juli 2019 (zur Publikation vorgesehen) E. 7.3.

17 Ebenda, E. 8.1.

18 Ebenda, E. 8.1. 
systemen weniger Gewicht beizumessen als in gemischten Systemen. Abweichungen seien dann gerechtfertigt, wenn die Bedeutung der Parteizugehörigkeit offenkundig in den Hintergrund rücke. ${ }^{19}$ Dies sei vermutungsweise in allen Wahlkreisen ausser den sechs grössten mit über 7000 Schweizer Einwohnern der Fall. ${ }^{20}$ In den sechs grössten Wahlkreisen könne nicht davon ausgegangen werden, dass die Kandidierenden einer Mehrzahl von Wählerinnen persönlich bekannt seien, womit die Anwendung des Majorzsystems in diesen Wahlkreisen verfassungswidrig sei. ${ }^{21}$

7 Nicht gefolgt ist das Bundesgericht der Argumentation der Beschwerdeführer, es seien Einerwahlkreise einzuführen. Mit einer solchen Vorschrift würde zu stark in die kantonale Autonomie eingegriffen, wofür keine gesetzliche oder verfassungsrechtliche Grundlage bestehe. ${ }^{22}$

\section{Würdigung}

\section{Verfassungspolitisches Vorspiel}

8 Die Bündner Kantonsverfassung wurde zu Beginn des 21. Jahrhunderts totalrevidiert und 2004 vom Bund gewährleistet. 23 Bereits damals äusserte der Bundesrat Bedenken, dass das Wahlsystem für den Grossen Rat im Lichte der Wahlrechtsgleichheit problematisch sei. Nichtsdestotrotz wurde die Kantonsverfassung gewährleistet, weil eine Praxisänderung nicht ohne Vorankündigung vorgenom-

19 Ebenda, E. 8.2.

20 Ebenda, E. 8.4.

21 Ebenda, E. 8.5.

22 Ebenda, E. 8.6; auf diese Problematik wird im Folgenden nicht weiter eingegangen.

23 Bundesbeschluss über die Gewährleistung der Verfassung des Kantons Graubünden vom 15. Juni 2004 (BBl 2004 3643), 3643 Art. 1. men werden sollte. ${ }^{24}$ Zur Botschaft des Bundesrats äusserte sich insbesondere die Staatspolitische Kommission des Ständerats kritisch und betonte die weitgehende Autonomie der Kantone bei der Ausgestaltung ihres Wahlsystems. ${ }^{25}$ Hierzu ist zu bemerken, dass das Bündner Volk bereits mehrmals zur Frage Stellung genommen hatte, ob es ein Majorz- oder ein Proporzsystem bevorzuge. Die Entscheidung fiel dabei immer zugunsten des Majorzsystems aus. ${ }^{26}$ Die Bundesversammlung hütete sich also damals davor, gegenüber Graubünden ein Majorzverbot auszusprechen. ${ }^{27}$ Vor diesem Hintergrund ist auch das Urteil des Bundesgerichts durchaus brisant. Zwar spricht es kein Majorzverbot aus, doch stellt sich das Bundesgericht gegen den Willen der Bündner Bevölkerung, das bestehende Wahlsystem beizubehalten.

9 Zwischen der Gewährleistung der Bündner Kantonsverfassung 2004 und der Einreichung der vorliegenden Beschwerde ergingen verschiedene Urteile des Bundesgerichts zu kantonalen Wahlsystemen, namentlich zu Proporzsystemen und zu Mischsystemen, nicht aber zu reinen Majorzsystemen, wie es neben Grau-

24 Botschaft über die Gewährleistung der Verfassung des Kantons Graubünden vom 5. März 2004 (BBl 2004 1107), 1114 f. Ziff. 3.4.

25 Bericht der Staatspolitischen Kommission des Ständerates vom 24. Mai 2004, Kantonsverfassung Graubünden. Gewährleistung (BBl 2004 3635), 3637 f. Ziff. 3.

26 Christina Bundi Caldelari/Christian Rathgeb, Kritische Bemerkungen zur Gewährleistung der Bündner Kantonsverfassung vom 18. Mai 2003/ 14. September 2003, in: ZGRG 3/04, 92-100, 93 f. m.w.H.

27 Bei der Gewährleistung ging es lediglich um die Frage der Zulässigkeit von Majorzwahlsystemen im Allgemeinen. Nicht zur Debatte stand damals die konkrete Ausgestaltung des Wahlsystems (vgl. dazu unten Rz 12). 
bünden nur noch der Kanton Appenzell I.Rh. kennt. ${ }^{28}$ Das Bundesgericht bildete die Kriterien für kantonale Parlamentswahlverfahren so stark fort, dass zahlreiche Kantone ihre Wahlverfahren anpassen mussten oder dies im Lichte der neuen Rechtsprechung freiwillig taten. Die Rechtsprechung erfuhr teils starke Kritik, mitunter vonseiten der Kantone. 29 Die Kritik manifestierte sich schliesslich in der Einreichung zweier Standesinitiativen, ${ }^{\circ}$ die die Freiheit der Kantone in der Festlegung ihrer Wahlsysteme ausdrücklich in die Bundesverfassung schreiben wollten. ${ }^{31}$ Wären diese Initiativen nicht in der Schlussabstimmung abgelehnt worden, ${ }^{32}$ hätte das Bündner Wahlsystem in der aktuellen Form mit grosser Wahrscheinlichkeit überlebt und die vorliegende Beschwerde wäre abgewiesen worden.

$28 \overline{\text { Zum Wahlsystem des Kantons Appenzell I.Rh. }}$ vgl. Christoph Raess, Sind die Wahlsysteme der Kantone Graubünden, Appenzell I.Rh. und Uri verfassungskonform?, in: ZGRG 3/16, 98-111, $103 \mathrm{f}$.

29 Vgl. Bericht der Staatspolitischen Kommission des Ständerats zu den Standesinitiativen Zug und Uri vom 16. November 2017 (BBl 2018 1), 3 f.; ebenfalls kritisch: Giovanni Biaggini, Majorz und majorzgeprägte Mischsysteme: Parlamentswahlverfahren mit Verfalldatum?, in: $\mathrm{ZBl}$ 8/2016, 409-429, 413, 416; sowie Georg Müller, Gerichtsrubrik, Sind Wahlen von Parlamenten nach dem Majorzsystem verfassungswidrig?, in: SJZ 111/2015, 103-105, 105.

30 Standesinitiative Zug (14.307), Wiederherstellung der Souveränität der Kantone bei Wahlfragen. Änderung der Bundesverfassung; Standesinitiative Uri (14.316), Souveränität bei Wahlfragen.

31 Andreas Glaser, Kantonales Wahlrecht zwischen Proporz und Spielräumen, in: ders. (Hrsg.), Das Parlamentswahlrecht der Kantone, Zürich/ St. Gallen 2018, 287-303, Rz 23 ff.; mithin wäre bei Annahme des neuen Verfassungstextes erstmals explizit in der BV verankert gewesen, dass ein Verhältnis-, ein Mehrheitswahlverfahren oder eine Mischform verfassungskonform sind.

32 Schlussabstimmung $\mathrm{zu}$ den Standesinitiativen 14.307 und 14.316 (AB 2018 N 2251).
10 Es ist also zu vermuten, dass das verfassungspolitische Vorspiel der letzten zwei Jahre einen Einfluss auf den Entscheid hatte, auch wenn dieses im Urteil keine Erwähnung findet. Im Vergleich zu den vorangehenden Entscheiden auferlegt sich das Bundesgericht denn auch eine gewisse Zurückhaltung. Dies ist richtig, denn besonders bei kantonalen Wahlverfahren sollen die kantonalen Eigenheiten Eingang in die Ausgestaltung der Wahlsysteme finden können.33 Das Bundesgericht hat lediglich die Schranken der Verfassungsmässigkeit festzustellen - und nicht mehr. 34

11 Vor diesem Hintergrund ist zu begrüssen, dass den genannten Standesinitiativen keine Folge geleistet wurde. Die Verfassungsänderung wäre schliesslich nur für wenige Kantone von Relevanz gewesen, da die restlichen Kantone ihre Wahlsysteme bereits überarbeitet haben. $35 \mathrm{Ihr}$ Ziel, die extensive Rechtsprechung des Bundesgerichts einzudämmen, scheinen die Initiativen dennoch nicht komplett verfehlt zu haben.

\section{Zulässigkeit der Überprüfung der Kantonsverfassung}

12 Das Bundesgericht überprüfte im vorliegenden Entscheid Art. 27 Abs. 2 KV/GR und führte damit eine inzidente Normenkontrolle durch. Dies durfte es nur

33 Auch die Ausführung, «dass vielen Anliegen, welche zur Rechtfertigung des Majorzsystems vorgebracht werden, mit entsprechenden Vorkehrungen auch im Proporzwahlverfahren Rechnung getragen werden kann» (BGE 143 I 92 E. 6.2, S. 103), unterlässt das Bundesgericht und respektiert damit den Entscheid des Kantons zugunsten eines Majorzsystems.

34 Ähnlich auch Yvo Hangartner, Die Wahl kantonaler Parlamente nach dem Majorzsystem, in: ZBl 106/2005, 217-233, 228.

35 Vgl. Glaser (Fn. 31), Rz 34 ff. 
deshalb, weil sich seit der Gewährleistung durch den Bundesgesetzgeber einerseits die Gegebenheiten im Kanton (Gebietsreform) ${ }^{36}$ geändert hatten, und andererseits, weil es seither die Kriterien zu den Wahlsystemen in verschiedenen Urteilen signifikant fortgebildet hatte. 37 Allerdings ist festzuhalten, dass der vorliegende Entscheid wohl auch ohne die Normenkontrolle gleich ausgefallen wäre. Der einschlägige Art. 27 Abs. 2 KV/GR sieht nämlich lediglich vor, dass für die Parlamentswahlen das Mehrheitswahlverfahren anzuwenden ist. Die grundsätzliche Verfassungsmässigkeit von Majorzwahlen hat das Bundesgericht richtigerweise nicht infrage gestellt. $3^{8}$ Vielmehr ist es die konkrete Ausgestaltung der Wahlen und diese wird auf Gesetzesstufe geregelt -, die das Bündner Wahlsystem verfassungswidrig macht.

${ }_{36} \overline{\mathrm{Zu} \text { einer Erläuterung der Gebietsreform s. Raess }}$ (Fn. 28), 100 f. Die Gebietsreform per 1. Januar 2016 führte zu einer grundlegenden Neuorganisation der Gebietsstrukturen in Graubünden. Während der Kanton früher in Gemeinden, Kreise, Bezirke und Regionalverbände eingeteilt war, bestehen neu nur noch Gemeinden und elf Regionen (Art. 68 Abs. $1 \mathrm{KV} / \mathrm{GR}$ ). Die Kreise haben damit ihre ursprüngliche Bedeutung verloren und dienen nur noch als Wahlkreise. Ebenfalls zu beachten gilt es, dass die Gemeindestrukturen durch zahlreiche Gemeindefusionen laufend verändert werden (hierzu: Amt für Gemeinden Graubünden, Entwicklung der Anzahl Gemeinden Graubündens).

37 Urteil des Bundesgerichts 1C_495/2017 vom 29. Juli 2019 (zur Publikation vorgesehen) E. $5.2 \mathrm{f}$.

38 Kritisch zu Majorzsystemen äussern sich u.a. Auer (Fn. 2), Rz 1143 ff.; ders., Majorz oder Proporz?, in: Christian Rathgeb et al. (Hrsg.), Graubündens Weg in die Zukunft, Zur Entwicklung und Erneuerung des bündnerischen Verfassungsrechts, Chur 2010, 54-56; Andrea Töndury, Die «Proporzinitiative 2014» im Kanton Graubünden, in: ZGRG 2/12, 64-86; Julian Marbach, Ist der Majorz für kantonale Parlamentswahlen verfassungsgemäss?, in: Jusletter 28. November 2016.

\section{Die Hauptprobleme des Bündner Wahlsystems}

\section{a) Verletzung der Stimmkraftgleichheit}

13 Probleme mit der Stimmkraftgleichheit treten dort auf, wo unterschiedlich grosse Wahlkreise gebildet werden. Dabei sind die grössten Ungleichheiten in der Stimmkraft in der Regel Sitzgarantien zuzuschreiben. Aber auch bei Wahlkreisen, die mehr als einen Sitz zu vergeben haben, können teils erhebliche Ungleichheiten entstehen. ${ }^{39}$ In seinem Entscheid geht das Bundesgericht überwiegend auf die Verzerrungen durch Sitzgarantien ein und erwähnt die mehrsitzigen Wahlkreise lediglich am Rande. ${ }^{40}$

14 Schon in früheren Urteilen akzeptierte das höchste Gericht Einschränkungen der Stimmkraftgleichheit bei Vorliegen sachlicher Gründe. ${ }^{41}$ Als sachlicher Grund wurde die Autonomie einer Gemeinde erachtet. $42 \mathrm{Ob}$ weitere sachliche Gründe eine Einschränkung rechtfertigen können, blieb bisher ungeklärt. Ebenfalls offen war, wo die Grenze der zulässigen Abweichungen von der Stimmkraftgleichheit liegt.43

15 Das Bundesgericht hält bezüglich der in Art. 2 Abs. 1 lit. a Ziff. 1 GRG/GR vorgesehenen Sitzgarantien fest, dass diese, anders als in den Fällen Uri und Schwyz, nicht dazu dienten, mit Autonomie ausgestatteten Gemeinden zu einer Repräsentation im Kantonsparlament zu ver-

39 Vgl. unten Rz 19 und Fn. 55.

40 Urteil des Bundesgerichts 1C_495/2017 vom 29. Juli 2019 (zur Publikation vorgesehen) E. 7.4.

41 BGE 143 I 92 E. 3.4, S. 95; BGE 125 I 21 E. 3d/dd, S. 33 .

42 BGE 143 I 92 E. 6.4, S. 108.

43 Ehrat/Eigenmann (Fn. 12), Rz 29 f. m.w.H. 
helfen. Es begründet dies damit, dass die Sitzgarantien in Graubünden zugunsten von Kreisen - Überbleibsel aus der Zeit vor der Gebietsreform - und nicht von Gemeinden gehen.44 Dies allein genügt gemäss Bundesgericht aber noch nicht, um die Sitzgarantien für verfassungswidrig zu erklären. Die Sitzgarantien sind im Ergebnis erst dann mit Art. 34 BV unvereinbar, wenn sie zu erheblichen Verzerrungen der Stimmkraftgleichheit führen.

Das Bundesgericht bringt fast unbemerkt eine neue Vorgabe ins Spiel: Als Richtwert sei für die minimale Wahlkreisgrösse in Majorzsystemen von der Hälfte der durchschnittlichen Repräsentationsziffer auszugehen.45 Es lässt für diesen Richtwert nicht nur eine Begründung vermissen, auch Bezüge auf Ansichten der Lehre sucht man vergebens. Diese Vorgabe ist erstaunlich zurückhaltend: Die Lehre akzeptierte bis anhin nur Abweichungen von bis $\mathrm{zu} 15 \%$ des mittleren Vertretungsanspruchs im Parlament. ${ }^{46}$ Bei der nun vom Bundesgericht gemachten Vorgabe resultieren zulässige Abweichungen von deutlich über $50 \% .47$

Die höchstrichterliche Kulanz geht sogar so weit, dass die Einteilung der Kreise Belfort und Rheinwald als verfassungs-

44 Vgl. Urteil des Bundesgerichts 1C_495/2017 vom 29. Juli 2019 (zur Publikation vorgesehen) E. 7.3.

45 Ebenda, E. 7.3.

46 Andrea Töndury, Der ewige K(r)ampf mit den Wahlkreisen, in: Andrea Good/Bettina Platipodis (Hrsg.), Direkte Demokratie, Herausforderungen zwischen Politik und Recht, Festschrift für Andreas Auer zum 65. Geburtstag, Bern 2013, 5169, 53; Thomas Poledna, Wahlrechtsgrundsätze und kantonale Parlamentswahlen, Diss. Zürich 1988, 94; allgemein zur Stimmkraftgleichheit vgl. Ehrat/Eigenmann (Fn. 12), Rz 28 ff.

47 Dazu die Berechnung zum Wahlkreis Belfort mit 665 Einwohnern bei Ehrat/Eigenmann (Fn. 12), 199. konform akzeptiert wird, obwohl sie (wenn auch nur sehr knapp) unter dem vorgegebenen Richtwert zu liegen kommen.48 Dies aufgrund des «Umstands, dass die historisch gewachsene Wahlkreiseinteilung nicht ohne Vorliegen gewichtiger Gründe als verfassungswidrig bezeichnet werden kann, sowie aus Gründen der Praktikabilität»49. Aber ist der Umstand, dass mit der Gebietsreform genau diese historisch gewachsenen Kreise abgeschafft wurden, nicht gerade solch ein gewichtiger Grund? Beim Wahlkreis Avers jedenfalls brachte das Bundesgericht gerade dieses Argument zur Begründung vor.50 Dass dem Bundesgericht bei seiner Argumentation nicht ganz wohl ist, wird am anschliessenden obiter dictum ersichtlich: Wenn sich die Einwohnerzahl in den Wahlkreisen Belfort und Rheinwald deutlich verringern sollte und sie damit deutlich kleiner als die durchschnittliche Repräsentationsziffer würde, wären auch diese Wahlkreise nicht mit Art. 34 Abs. 2 BV vereinbar. ${ }^{51}$ Aber selbst für solche Fälle bringt das Bundesgericht den Vorbehalt an, dass in besonderen Situationen und bei Vorliegen gewichtiger Gründe ein Wahlkreis dennoch verfassungskonform sein könnte. $5^{2}$ Damit wird die verfassungsrechtliche Grenze für die minimale

$4 8 \longdiv { \text { Urteil des Bundesgerichts 1C_495/2017 vom } }$ 29. Juli 2019 (zur Publikation vorgesehen) E. 7.3.

49 Ebenda, E. 7.3; auffallend an dieser Begründung ist auch, dass das Bundesgericht bis anhin das historische Argument für eine Abweichung von der Stimmkraftgleichheit noch nie erwähnt hat. Vielmehr wurde bis anhin nur die Autonomie von Wahlkreisen als möglicher Rechtfertigungsgrund erwähnt (dazu Ehrat/Eigenmann [Fn. 12], Rz 30; BGE 143 I 92 E. 6.4, S. 108).

50 Urteil des Bundesgerichts 1C_495/2017 vom 29. Juli 2019 (zur Publikation vorgesehen) E. 7.3.

51 Ebenda, E. 7.3.

52 Ebenda, E. 7.3 in fine. 
Wahlkreisgrösse freilich noch weiter nach unten geschraubt.

Somit gilt für Majorzsysteme (neu): Sitzgarantien sind verfassungskonform, auch wenn sie nicht dazu dienen, mit Autonomie ausgestattete Gemeinden im Parlament zu repräsentieren. Verfassungswidrig sind sie erst dann, wenn sie den Richtwert von Abweichungen von $50 \%$ von der durchschnittlichen Repräsentationsziffer deutlich überschreiten - vorbehältlich «besondere[r] Situationen, in denen gewichtige sachliche Gründe ausnahmsweise für die Beibehaltung eines bestimmten Wahlkreises trotz zu geringer Wohnbevölkerung sprechen würden»53. Fraglich ist im Lichte dieser neuen Rechtsprechung, bis zu welcher Grenze das Bundesgericht künftig Abweichungen vom Richtwert bei Wahlkreisen, die sich auf ihre Autonomie oder andere gewichtige sachliche Gründe berufen können, akzeptieren wird.

Es ist zu bedauern, dass der vom Bundesgericht aufgestellte Richtwert derart hoch ist. Auch wenn die exzessivsten Abweichungen der Stimmkraft für den Wahlkreis Avers (zwölffache Stimmkraft!) verhindert wurden, bleibt die Stimmkraft einer Wählerin beispielsweise in Belfort fast dreimal so gross wie diejenige eines Wählers in Churwalden. Es ist nicht ersichtlich, warum Verzerrungen der Stimmkraftgleichheit in so hohem Masse auch bei Wahlkreisen hinzunehmen sind, die sich nicht auf eine besondere Autonomie stützen können und teilweise sogar Gegenstand von Gerrymandering54 werden. Dazu kommt,

53 Ebenda, E. 7.3.

54 Vgl. zu den taktisch motivierten Wahlkreisverschiebungen in der Surselva: La Surselva vul dass auch in mehrsitzigen Wahlkreisen erhebliche Ungleichheiten resultieren können, ohne dass diese auf Sitzgarantien zurückzuführen wären.55 Diese Problematik hat das Bundesgericht entweder ausser Acht gelassen, oder aber einfach akzeptiert. ${ }^{6}$ Es wäre wünschenswert gewesen, hätte sich das Bundesgericht für die Festsetzung der maximal zulässigen Verzerrungen der Stimmkraft an der Lehre57 orientiert. Freilich dürfte es sich dabei nicht um starre Grenzen handeln. Vielmehr muss anhand des jeweiligen Einzelfalls eruiert werden, ob eine stärkere Verzerrung aufgrund sachlicher Gründe zu rechtfertigen ist.

20 Ebenfalls unterliess es das Bundesgericht in Erwägung zu ziehen, dass seit der Gebietsreform eine Sitzgarantie auf Verfassungsstufe nicht mehr vorgeschrieben ist (Art. 27 Abs. 3 KV/GR e contrario). Die Kantonsverfassung schreibt nur noch vor, dass höchstens 39 Wahlkreise gebildet werden können. Auch das deutet daraufhin, dass die Autonomie der einzelnen Wahlbezirke weiter an Bedeutung verliert und eine einfachere Neueinteilung der Wahlkreise ermöglicht werden sollte.

mantegnair ils 14 sezs da deputads; vgl. Ehrat/ Eigenmann (Fn. 12), Rz 45.

55 Dazu können die Wahlkreise Misox (1998 Einwohner, 2 Sitze) und Churwalden (1918 Einwohner, 1 Sitz) beispielhaft angeführt werden (vgl. Ehrat/Eigenmann [Fn. 12], 199). Die Stimme einer Wählerin in Misox wiegt doppelt so schwer wie diejenige einer Churwaldnerin.

56 Vgl. Urteil des Bundesgerichts 1C_495/2017 vom 29. Juli 2019 (zur Publikation vorgesehen) E. 7.4, wo das Bundesgericht die mehrsitzigen Wahlkreise zwar anspricht, jedoch nur auf zulässige Rundungsabweichungen eingeht. Von einfachen Rundungsabweichungen kann im soeben gemachten Beispiel (vgl. Fn. 55) nicht die Rede sein.

57 Vgl. oben Rz 16. 


\section{b) Verletzung der Erfolgswertgleichheit}

Bisher pochte das Bundesgericht trotz Einwänden aus der Lehre58 darauf, dass die Erfolgswertgleichheit als Teilgehalt der Wahlrechtsgleichheit auch bei Majorzwahlverfahren zu beachten ist. Diese Rechtsprechung führt es im vorliegenden Urteil weiter und überträgt sie mit gewissen Einschränkungen auf reine Majorzsysteme.

Das Bundesgericht beschäftigt sich in seinen Überlegungen zur Erfolgswertgleichheit überwiegend mit der Bedeutung der Parteizugehörigkeit in Majorzsystemen. Mithin sei die Parteizugehörigkeit auch in Majorzwahlverfahren von Bedeutung. Vor diesem Hintergrund erachtet das Bundesgericht die bei Majorzsystemen auftretende ungenaue Abbildung der Parteistärken im Parlament als problematisch.59 Allerdings sieht es die Einschränkung der Erfolgswertgleichheit in Majorzwahlkreisen weiterhin als zulässig an, wenn die Persönlichkeit und nicht die Parteizugehörigkeit der Kandidierenden für die Wahl ausschlaggebend ist. In solchen Fällen «können die Vorteile des Majorzprinzips grösser sein als seine Nachteile»60; dann trete auch das Gebot der proportionalen Abbildung im Parlament in den Hintergrund. Dies sei dann wahrscheinlicher, wenn es sich um kleine Wahlkreise handelt, wenn sich das politische und gesellschaftliche Leben dezentralisiert abspielt und wenn Wähler und Kandidaten stark in ihrem Wahl-

${ }_{58} \mathrm{Vgl}$. die vertiefte Auseinandersetzung mit diesem Problem in Biaggini (Fn. 29), 412 ff. bezüglich Majorzsystemen, $420 \mathrm{ff}$. bezüglich Mischsystemen.

59 Urteil des Bundesgerichts 1C_495/2017 vom 29. Juli 2019 (zur Publikation vorgesehen) E. 8.1.

6o Ebenda, E. 8.1. kreis verwurzelt sind. ${ }^{61}$ Diese Kriterien sind bekannt und wurden - wie erwartet - von der Rechtsprechung zu Mischsystemen auf die neue Rechtsprechung zu reinen Majorzsystemen übertragen.

Zusätzlich lässt es das Bundesgericht nun $\mathrm{zu}$, dass bei reinen Majorzsystemen die Erfolgswertgleichheit noch weiter in den Hintergrund tritt. ${ }^{62}$ Dies rechtfertige sich vor dem Hintergrund des bewussten Entscheids des Kantons gegen ein Verhältniswahl- oder Mischsystem. In solchen Fällen genüge es bereits, wenn zumindest wahrscheinlich ist, dass die Zugehörigkeit zu einer Partei nicht zu stark im Vordergrund steht, sondern vielmehr die Persönlichkeit des Kandidaten von einer gewissen Bedeutung ist. ${ }^{63}$ Nach Ansicht des Bundesgerichts rechtfertigt sich ein reines Majorzsystem also nur dann nicht, wenn die Parteizugehörigkeit aus Sicht der Wählenden eine offensichtlich dominierende Rolle spielt. Als Grund für dieses weitere Zurücktreten der Erfolgswertgleichheit führt das Bundesgericht nota bene die Beachtung der kantonalen Autonomie nach Art. 39 Abs. 1 BV an und nicht den Umstand, dass bei Majorzsystemen die Erfolgswertgleichheit schlicht nicht verwirklicht werden kann.

24 Das ebengenannte Kriterium sah das Bundesgericht mit den Beschwerdegegnern für die Wahlkreise mit weniger als 7000 Einwohnern vermutungsweise als erfüllt an und führte ohne weitere Begründung an, dass daran auch die inzwischen beseitigte Autonomie der Wahlkreise nichts zu ändern vermöge. ${ }^{64}$ Es

\footnotetext{
61 Ebenda, E. 8.1.

62 Ebenda, E. 8.2.

63 Ebenda, E. 8.2.

64 Ebenda, E. 8.4.
} 
wird hier also eine starre Grenze für die Vermutung der Bedeutungslosigkeit der Parteizugehörigkeit eingeführt.

Problematisch ist das Kriterium der fehlenden Bedeutung der Parteizugehörigkeit auf einer faktischen Ebene. ${ }^{65}$ Zwar begnügt sich das Bundesgericht weiterhin mit nachvollziehbaren Behauptungen, dass die Parteizugehörigkeit von Kandidaten nur eine untergeordnete Rolle spielt. ${ }^{66}$ Und dies, obwohl die Gebietsreform und die wenigen parteilosen Kandidaten eher den gegenteiligen Schluss nahelegen würden. Doch ist unklar, wie das Bundesgericht dieses Problem künftig in grösseren Wahlkreisen handhaben wird, wo eigentlich eine vertiefte Prüfung der Verhältnisse nötig wäre. Dieses Problem akzentuiert sich weiter, wenn man bedenkt, dass das Bundesgericht dem Kriterium der fehlenden Autonomie der Wahlkreise in diesem Entscheid praktisch keine Bedeutung mehr zumisst. ${ }^{6} 7$ Die Frage nach der Bedeutung der Parteizugehörigkeit einer Kandidatin wird damit zum eigentlichen Gradmesser für die Zulässigkeit von Majorzwahlsystemen. Vor dem Hintergrund der schwierigen Überprüfbarkeit dieses Kriteriums

65 Dazu Glaser (Fn. 31), Rz 26; es ist daran zu erinnern, dass das Bundesgericht das Kriterium im Urner und Ausserrhoder Entscheid noch explizit als Tatfrage bezeichnete (BGE 143 I 92 E. 6.3.4, S. 106; Urteil des Bundesgerichts 1C_59/2012, 1C_61/2012 vom 26. September 2014 E. 12.5.1 [nicht publ. in BGE 140 I 93]). Auf diese Ausführungen verzichtet das Bundesgericht vorliegend.

66 Urteil des Bundesgerichts 1C_495/2017 vom 29. Juli 2019 (zur Publikation vorgesehen) E. 8.1 ff.; vgl. auch BGE 143 I 92 E. 6.3.4, S. 106.

67 In früheren Entscheiden kam diesem Kriterium bereits nur untergeordnete Bedeutung zu: vgl. Urteil des Bundesgerichts 1C_59/2012, 1C_61/2012 vom 26. September 2014 E. 12.6 (nicht publ. in BGE 140 I 93); vgl. Musliu (Fn. 10), Rz 62. leidet mit dieser Rechtsprechung im Ergebnis die Rechtssicherheit. 68

26 Es kann festgehalten werden: Bei reinen Majorzsystemen hat die Erfolgswertgleichheit einen noch kleineren Stellenwert als bei Mischsystemen, die ihrerseits eine Abweichung von der Erfolgswertgleichheit einfacher rechtfertigen können als Proporzsysteme. Zulässig ist ein Majorzsystem, solange nicht offensichtlich ist, dass die Parteizugehörigkeit der Kandidierenden eine vordergründige Bedeutung für die Wähler hat. Dies ist bei Wahlkreisen mit weniger als 7000 Einwohnern zu vermuten. Bei grösseren Wahlkreisen ist hingegen vom Gegenteil auszugehen: Der Nachweis der fehlenden Bedeutung der Parteizugehörigkeit dürfte hier regelmässig misslingen. Dieser Rechtsprechung ist im Ergebnis zuzustimmen, auch wenn das Abstellen auf das Kriterium der Parteizugehörigkeit aus den genannten Gründen nicht unproblematisch ist. Die Vermutung der Verfassungskonformität von Majorzwahlkreisen mit höchstens 7000 Einwohnern ist zwar klar und praktikabel, weil dadurch aktuell keine problematischen Grenzfälle entstehen. Gerade deshalb wirkt der Grenzwert aber zufällig und dürfte darüber hinaus keine Bedeutung haben.

\section{Mögliche Folgen des Entscheids}

27 Nach dem vorliegenden Urteil bleibt der Bündner Regierung nicht viel Zeit, um ein neues Wahlsystem auszuarbeiten. Bereits im Jahr 2022 stehen die nächsten Gesamterneuerungswahlen an; in Anbetracht dessen wurde im Grossen Rat be-

68 Dazu Biaggini (Fn. 29), 420. 
reits ein erster Vorstoss eingereicht. 69 Die Aufgabe der Regierung und des Grossen Rats ist mitnichten einfach, denn es kommen verschiedene Ausgestaltungen für das neue Wahlsystem infrage.

Möglich und wahrscheinlich scheint die Einführung eines gemischten Wahlsystems, wie es die Kantone Appenzell A.Rh. und Uri bereits kennen. Als Massstab könnten die Urteile des Bundesgerichts zu diesen Kantonen dienen. ${ }^{70}$ Nicht ignorieren sollte die Bündner Regierung dabei die aktuellen Entwicklungen rund um das Urner Wahlsystem: Nachdem das Bundesgericht dieses 2016 als verfassungswidrig beurteilt hatte, beschloss der Kanton Uri am 19. Mai 2019 eine neue Wahlordnung. Einerseits wurde für die Proporzgemeinden auf Gesetzesstufe der «Doppelte Pukelsheim»71 eingeführt, andererseits wurde überraschenderweise die Anzahl der im Majorz wählenden Wahlkreise erhöht. Neu findet das Majorzsystem nicht mehr nur auf Wahlkreise mit bis zu zwei Sitzen Anwendung, sondern auf Wahlkreise mit bis zu vier Sitzen. Hierfür war eine Verfassungsänderung notwendig; 72 mit Spannung ist daher der Gewährleistungsentscheid des Bundesgesetzgebers $\mathrm{zu}$ erwarten, der nicht nur für die Kantone, sondern auch für das Bundesgericht wegweisend sein wird. Insbesondere wird interessant sein,

$69 \overline{\text { Auftrag Claus betreffend Anpassung des Wahlsys- }}$ tems für den Grossen Rat vom 29. August 2018.

70 BGE 140 I 394; BGE 143 I 92.

${ }^{71}$ Dazu Friedrich Pukelsheim, Sitzzuteilungsmethoden, Ein Kompaktkurs über Stimmenverrechnungsverfahren in Verhältniswahlsystemen, Berlin 2016, $85 \mathrm{ff}$.

72 Abstimmungsbotschaft zur Änderung des Gesetzes über die Verhältniswahl des Landrats (Proporzgesetz) und zur Änderung der Verfassung des Kantons Uri. wie der Bundesgesetzgeber bei dieser Gelegenheit zum vorliegenden Urteil Stellung beziehen wird.73

29 Möglich, aber unwahrscheinlich ist der Übergang zu einem Verhältniswahlverfahren. Dazu müsste Graubünden eine grundlegende Neueinteilung seiner Wahlkreise vornehmen. Bei einer Einführung des Proporzsystems müsste die Rechtsprechung des Bundesgerichts beachtet werden. Diese akzeptiert natürliche Quoren von maximal 10\% - jeder Wahlkreis braucht also mindestens neun Sitze.74 Die Wahlkreisgrösse, die das Bundesgericht vorliegend unter Majorzgesichtspunkten als verfassungsmässig betrachtete, wäre in einem Proporzwahlsystem verfassungswidrig, solange keine ausgleichenden Massnahmen wie Wahlkreisverbände vorgesehen würden. Die grösste Hürde wäre bei Einführung eines Proporzsystems aber wahrscheinlich das Bündner Volk, das sich bisher stets gegen ein Proporzwahlsystem gestellt hat.75

30 Die Beibehaltung eines reinen Majorzsystems dürfte schwierig zu rechtfertigen sein. Selbst wenn die grössten Wahlkreise verkleinert bzw. aufgeteilt würden, ${ }^{76}$ würde dies nichts an der vorherrschenden Bedeutung der Parteizugehörigkeit ändern. Denn selbst wenn eine Stadt wie Chur auf mehrere Wahlkreise aufgeteilt wird, dürfte die Parteizugehörigkeit der

$73 \overline{\text { Der Bundesrat hat bereits zur Verfassungsände- }}$ rung Stellung genommen: Botschaft zur Gewährleistung der geänderten Verfassungen der Kantone Uri, Tessin, Waadt, Wallis und Genf vom 6. Dezember 2019 (BBl 2020 147), 150 ff. Ziff. 1.1.3 f.

74 BGE 131 I 74 E. 5.4, S. 83; dazu Weber (Fn. 10), Rz 312.

75 Vgl. dazu oben Rz 8 und Fn. 26.

76 Vgl. dazu die Ausführungen des Bundesgerichts: Urteil des Bundesgerichts 1C_495/2017 vom 29. Juli 2019 (zur Publikation vorgesehen) E. 8.5.3. 
Kandidaten dort einen überwiegenden Einfluss auf die Wahl beibehalten. Dies zeigt eine weitere Problematik des Kriteriums der Bedeutung der Parteizugehörigkeit auf. Eine Aufteilung der Wahlkreise würde bei Wahlkreisen mit über 7000 Einwohnern nicht zwingend zur Verfassungskonformität führen.

Welchen Weg Graubünden auch einschlägt - eine Neueinteilung der Wahlkreise ist für ein seriöses Wahlsystem Bedingung. Wie das Bundesgericht zutreffend feststellt, entspricht die heutige Einteilung längst nicht mehr einer historischen Gebietsorganisation. Dies ist einerseits der unlängst vorgenommenen Gebietsreform und andererseits dem in gewissen Kantonsteilen praktizierten Gerrymandering 77 zuzuschreiben. Die aufgezwungene Wahlreform muss Graubünden als Anlass nehmen, seine Gebietsstrukturen sorgfältig zu analysieren und das Wahlsystem daran anzupassen nicht umgekehrt. Nicht ohne Grund lautete das Motto der Gebietsreform ursprünglich: «zuerst Gebiets- und dann Wahlreform» 78 . Bei der Wahlkreisreform ist auf eine Mischung zwischen proportionaler Repräsentation und Berücksichtigung von sprachlichen und kulturellen Gegebenheiten zu achten.

Interessant ist auch die Frage, welche Implikationen dieses Urteil für den Kanton Appenzell I.Rh. haben wird, dem einzigen anderen Majorzkanton. Dessen Wahlsystem war bisher wohl als gerade noch verfassungskonform zu betrachten.

Vgl. dazu oben Fn. 54 .

78 Botschaft der Regierung an den Grossen Rat, Heft Nr. 8/2010-2011, 589-706, 670; die Regierung änderte ihre Meinung allerdings später: Botschaft der Regierung an den Grossen Rat, Heft Nr. 10/2013-2014, 757-842, 772.
Dies könnte sich allerdings ändern, sobald die Parteizugehörigkeit der Kandidierenden an Bedeutung gewinnt.79 Daher erscheint es wahrscheinlich, dass das Bundesgericht in naher Zukunft auch zu Appenzell I.Rh. eine Beschwerde zu beurteilen haben wird - sofern ihr der Kanton nicht mit einer eigenen Wahlreform zuvorkommt.

\section{Schlussbetrachtung}

33 Das Bundesgericht war in seiner bisherigen Rechtsprechung zu gemischten Wahlordnungen der Ansicht, dass solche Systeme grundsätzlich besser geeignet sind, die verfassungsrechtlichen Vorgaben einzuhalten, als reine Majorzverfahren. ${ }^{80}$ Es brachte gegenüber Majorzsystemen gar eine grundsätzliche Abneigung zum Ausdruck. Von dieser wohl überschiessenden Rechtsprechung ist das Bundesgericht zwar nicht abgerückt, doch übt es in Bezug auf neue Vorgaben mehr Zurückhaltung. Diese Zurückhaltung vermag hinsichtlich der neu etablierten Massgaben allerdings nicht zu überzeugen, zumal erhebliche Verzerrungen der Stimmkraftgleichheit hingenommen werden und neue Rechtsunsicherheit geschaffen wird. Das Bundesgericht hätte auch ein härteres Verdikt zum Bündner Majorz fällen können, ohne sich dem Vorwurf einer exzessiven Rechtsfortbildung auszusetzen. Im Ergebnis hätte dies aber für das Bündner Wahlsystem wenig verändert. So oder anders ist es verfassungswidrig und muss durch ein modernes, den aktuellen Gegebenheiten Rechnung tragendes Wahlsystem ersetzt

79 Zum Ganzen Ehrat/Eigenmann (Fn. 12), Rz 59 ff., insb. $73 \mathrm{f}$.

8o BGE 143 I 92 E. 6.2, S. 102 f. 
werden. Die Folgen des Urteils sind daher trotz höchstrichterlicher Zurückhaltung weitreichend. 